\title{
Temporal Scalability through Adaptive $M$-Band Filter Banks for Robust H.264/MPEG-4 AVC Video Coding
}

\author{
C. Bergeron, ${ }^{1}$ C. Lamy-Bergot, ${ }^{1}$ G. Pau, ${ }^{2}$ and B. Pesquet-Popescu ${ }^{2}$ \\ ${ }^{1}$ EDS/SPM, THALES Communications, 92704 Colombes Cedex, France \\ ${ }^{2}$ TSI Department, Ecole Nationale Supérieure des Télécommunications, 75634 Paris Cedex 13, France
}

Received 15 March 2005; Revised 4 September 2005; Accepted 19 September 2005

This paper presents different structures that use adaptive $M$-band hierarchical filter banks for temporal scalability. Open-loop and closed-loop configurations are introduced and illustrated using existing video codecs. In particular, it is shown that the H.264/MPEG-4 AVC codec allows us to introduce scalability by frame shuffling operations, thus keeping backward compatibility with the standard. The large set of shuffling patterns introduced here can be exploited to adapt the encoding process to the video content features, as well as to the user equipment and transmission channel characteristics. Furthermore, simulation results show that this scalability is obtained with no degradation in terms of subjective and objective quality in error-free environments, while in error-prone channels the scalable versions provide increased robustness.

Copyright ( 92006 C. Bergeron et al. This is an open access article distributed under the Creative Commons Attribution License, which permits unrestricted use, distribution, and reproduction in any medium, provided the original work is properly cited.

\section{INTRODUCTION}

Modern wireless communication applications relying on the use of video services and video streaming are facing a problem that high-speed wired networks seemed to have overcome: for them, the available bandwidth is still a limiting factor. Moreover, IP wireless networks have to cope with both bit errors and packet losses. This is why a new generation of standards, such as H.264/MPEG-4 AVC finalized in May 2003 [1] jointly by ISO MPEG and ITU-T, and also the new wavelet-based codecs solutions proposed within the scalable video coding (SVC) group, such as [2], take into account the interaction with the network (for the former, through the network abstraction layer concept). Such codecs provide significant compression efficiency improvement when compared to the other existing standards such as MPEG-2 or MPEG-4; and that is why they are so attractive for multimedia applications over wireless communication links. However, H.264/MPEG-4 AVC does not support scalability, which is a very efficient tool to adapt to the bandwidth variations and to the error-prone nature of the wireless channels. Temporal scalability can be achieved using B frames in profiles that support them, which is not the case of H.264/MPEG-4 AVC baseline profile. Solutions are currently being proposed in the literature and within the SVC standardisation group to address this limitation, generally by introducing modifications to the H.264/MPEG-4 AVC syntax to integrate pro- gressive fine granular scalability coding or subband decompositions $[3,4]$. In parallel, solutions relying on motioncompensated (MC) spatio-temporal subband decompositions are being proposed, first with a classical dyadic subband decomposition [5], then by exploiting a nonlinear lifting implementation [6], and making use of efficient 3D entropy coding algorithms [7]. Such solutions are unfortunately not compliant with basic H.264/MPEG-4 AVC decoders and often introduce a higher level of complexity, which may not be acceptable for the use in small and cheap mobile equipments.

Following the approach initiated in [8] where the introduction of temporal scalable solutions fully compliant with H.264/MPEG-4 AVC has been proposed and interpreted in the framework of adaptive $M$-band hierarchical filter banks, in this paper we show that this framework can be further generalized to include dyadic temporal decompositions and also to introduce scalability inside both openloop and closed-loop temporal prediction structures. In particular, we show that the resulting hierarchical representation of H.264/MPEG-4 AVC frames inside a group of pictures (GOP) preserves the coding performance of the original nonscalable scheme in an error-free environment, and improves the subjective and objective qualities of the sequences transmitted over error-prone channels.

This paper is organized as follows. Section 2 introduces the proposed hierarchical filter bank structures and discusses their interest for video coding and scalability. In Section 3, an 


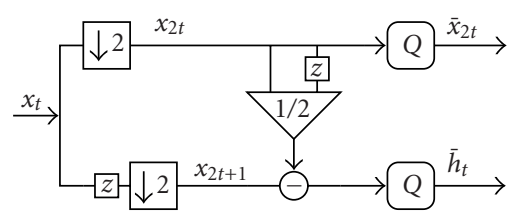

FIgURE 1: Open-loop prediction scheme: one level of decomposition.

application of these filter banks to the temporal prediction compliant with the H.264/MPEG-4 AVC standard is proposed and discussed. In Section 3.3 the adaptation of this filter bank scheme to the case of H.264/MPEG-4 AVC where the number of reference frames should be limited, namely, for simple levels, is considered. Section 4 describes a practical setup for easily applying filtering in a conformant way to an H.264/MPEG-4 AVC codec, through the application of an interleaver, as well as the simulation chain model considered for testing the various shuffling configurations, both in errorfree and error-prone environments. Finally, in Section 5 experimental results are presented and in Section 6 the conclusions are drawn.

\section{M-BAND HIERARCHICAL FILTER BANKS}

Temporal scalability is achieved by introducing a hierarchy among the frames encoded in a group of pictures. This is true for both classical closed-loop temporal differential pulse code modulation (DPCM) schemes, and for motion-compensated wavelet decompositions, using open-loop schemes based on motion-compensated temporal filter banks. In both cases, some constraints are introduced in the temporal prediction in order to create successive layers of importance. In this section we point out the analogies between the two approaches, by describing a common framework based on temporal subband decompositions.

Let us consider the lifting form of the motion-compensated wavelet decompositions [9]. Basically, the desired temporal dyadic filter bank is represented in its lifting form with one (or several) predict and update steps involving motion compensation. In designing these structures, particular attention should be paid to the motion prediction direction in the temporal operators so as to facilitate the filtering along motion trajectories. In order to simplify the comparison, our model will not include the update step (which is however essential for the good performances of these schemes). For a bidirectional prediction (from past and future frames, as commonly used in the 5/3 filter banks), the basic scheme is illustrated in Figure 1, where the input frames (at times $t \in \mathbb{N}$ ) are denoted by $x_{t}$, and the resulting temporal detail frames, corresponding to high temporal frequencies, are denoted by $h_{t}$. After the quantization block $Q$, the same frames are denoted by $\bar{x}_{t}$, respectively, $\bar{h}_{t}$. In this one-level decomposition, the even-indexed frames (following the notation in Figure 1) will enter the approximation subband, while the error prediction frames will yield the detail subband.

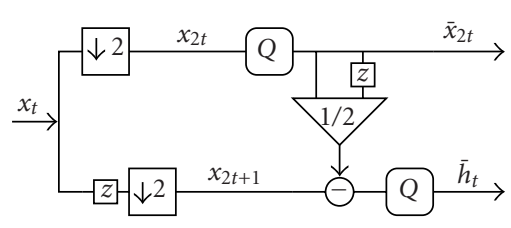

Figure 2: Basic closed-loop prediction scheme.

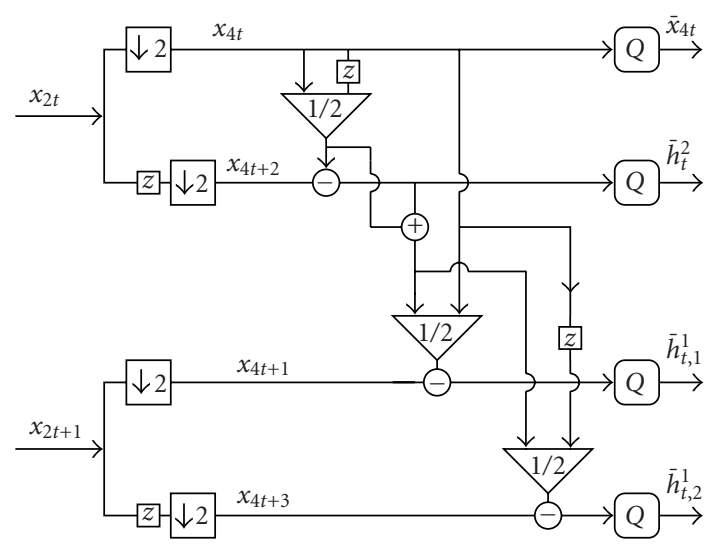

FIGURE 3: Open-loop scheme with 4 temporal subbands (2 temporal decomposition levels).

By just changing the place of one of the quantizers in Figure 1, we get a prediction based on the previously reconstructed frames, as illustrated in Figure 2 (here, for the sake of simplicity, the inverse quantization and the spatial direct and inverse transforms have been omitted).

By iterating the splitting into odd and even frames, we obtain a four-band polyphase decomposition, on which the successive application of the previous prediction scheme leads to an approximation subband (containing the equivalent to the intraframes), a detail subband at the coarse resolution level similar to a B frame in the base layer (denoted in Figure 3 by $h_{t}^{2}$ ), and two detail frames at the finest resolution level, $h_{t, 1}^{1}$ and $h_{t, 2}^{1}$, similar to B frames in the enhancement layer. Note that this hierarchical structure can be seen as consisting of two levels of a wavelet decomposition without the update lifting step.

The two-level structure in Figure 3 can be transposed into a closed-loop structure, equivalent to a four-band decomposition, as illustrated in Figure 4.

The previous open-loop and closed-loop subband decompositions can be extended to an arbitrary number of decomposition levels, involving groups of frames counting a power of two number of frames. ${ }^{1}$

\footnotetext{
${ }^{1}$ Note also that in [8] we have introduced temporal subband decompositions with an odd number of subbands, allowing pyramidal or treelike hierarchical structures.
} 


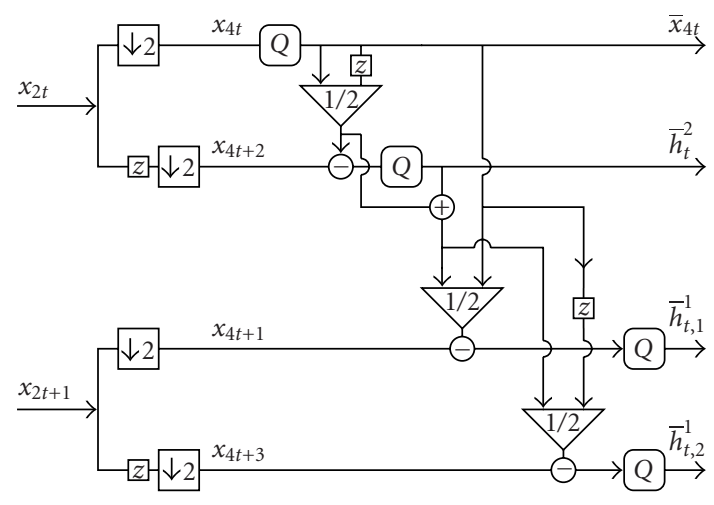

Figure 4: Closed-loop scheme with 4 temporal subbands (2 temporal decomposition levels).

A common property of these structures is that each GOP is independently decodable, which is a very useful feature in error-prone environments, in order to avoid error propagation.

\section{APPLICATION TO THE H.264/MPEG-4 AVC VIDEO STANDARD}

Relying on the motion-compensated temporal subband decompositions presented in Section 2, in this section we show that the existing properties of the H.264/MPEG-4 AVC standard allow us to build a hierarchical representation inside the GOPs without requiring any modification or addition to the standardized codec, thus remaining fully compliant with each of the standard profiles. ${ }^{2}$

Moreover, contrarily to previous video coding standards that were using simple reference modes, for which the prediction of interframes could only be done with respect to a given preceding picture, it is important to point out that H.264/MPEG-4 AVC allows the usage of up to 16 different frames as reference in some levels, for each P-slice. In practice, this capability means that several previous frames (in encoding order) can be used as references for the current frame.

Considering groups of pictures of $N$ frames, denoted by their original time reference $\{0,1,2, \ldots, N-1\}$, the aim of our approach is to intelligently distribute the frames so that the encoding process that will follow is done efficiently. As a matter of fact, the classical prediction order in the GOP may not be the most efficient one from a temporal scalability standpoint because (a) one wants to obtain a regular frame rate when using the temporal scaling, and (b) a better compression efficiency can be obtained when placing the reference frames closer to the predicted ones in display order [8]. The classical decomposition, which we call "Normal" con-

\footnotetext{
${ }^{2}$ In the baseline profile, this approach corresponds to using predictive (P) frames. But this method could also be easily generalized with $B$ frames for other profiles. As a consequence, the proposed temporal scalability feature is the only one compatible with all the profiles.
}

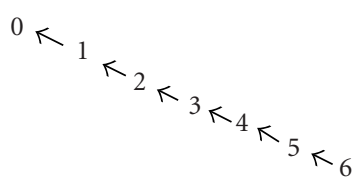

FIGURE 5: Normal configuration, GOP size $=7$. The arrows are directed towards the reference frame.

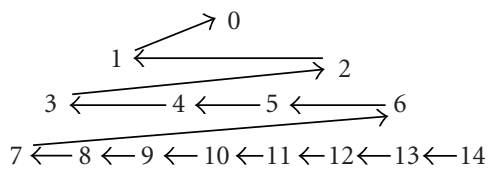

Figure 6: Zigzag configuration, GOP size $=15$.

figuration, is presented in Figure 5 in the case of a GOP size $N=7$. The dependencies between frames are illustrated by the arrows in the figure which shows that frame 1 depends on frame 0 , frame 2 on frame 1 and consequently also on frame 0 , and so forth. This can be obtained from the closed-loop scheme in Figure 4, by considering only unidirectional predictions and as many levels as the number of frames in the GOP.

Three different approaches for temporal scalability are considered in the following:

(i) symmetric filtering schemes, meaning that a unique intraframe, which is taken for these configurations to be actually an instantaneous decoding refresh (IDR) frame, is considered as a main reference for the whole GOP; in our approach by frame shuffling, it is placed in the middle of the GOP (in output display order);

(ii) asymmetric filtering schemes, where each intraframe is used as a reference by two consecutive GOPs;

(iii) a combination of the above two approaches, taking into account possible limits in terms of frame reference buffer sizes, to meet the eventually more restrictive requirements of certain levels of the standard and practical implementations.

\subsection{Symmetric filtering schemes}

A first decomposition configuration ensuring the temporal scalability features, that we will call "Zigzag" configuration, is illustrated in Figure 6 for $N=15$. Firstly introduced in [8], this regular pattern corresponds to the subband decomposition for GOP of size $N=2^{L}-1, L \in \mathbb{N}$. It is obtained as follows:

(i) select a reference frame (the intraframe) for the first level having the temporal index at the median value of the GOP, where the median index is median = $\left(\mathrm{GOP}_{\text {size }}+1\right) / 2$; define each part separated by the median as sub-GOP;

(ii) repeat for each sub-GOP: take as reference frames the median ones and define accordingly the remaining sub-GOPs for the next level. 


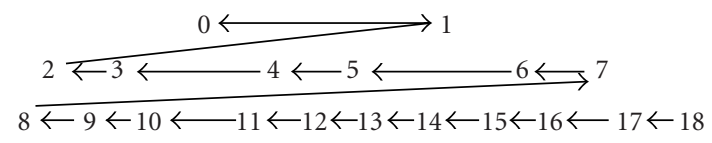

FIGURE 7: Generalized Zigzag configuration with $R=3$, GOP size $=$ 19.

In practice, one sees that the first resolution level consists only of the intraframe which is placed at the median value of the GOP (and not at the beginning of the GOP as usual). In this configuration, dependencies between frames are very important, as each frame $i$ can depend (based on the efficiency of the compression mechanism and of the considered sequence) on the $i-1$ previous ones. In practice, one observes that the coding efficiency is smaller for the first levels, since the temporal distance between the predicted and the reference frames can be greater than one. Still, simulation results show that this is in practice compensated by the fact that the latest frames offer better compression rate, as they are closer to their main reference frames.

This Zigzag decomposition is obviously very efficient for $N=2^{L}-1$, which corresponds to a fully regular repartition pattern of the subband decomposition, but can easily be used in other cases, at the cost of some loss in compression efficiency. In particular, one can think to generalize the decomposition to other subsampling factors $R$ (greater than 2), as well as for other values of $N$. This hierarchical structure can be achieved as follows [8]:

(i) select $R-1$ reference frames at each level (e.g., with the intraframe being the first of them) at equal temporal distance in the GOP, that is, having temporal indices $m_{i}=\left\lfloor i\left(\mathrm{GOP}_{\text {size }}+1\right) / R\right\rfloor$ for $i=1, \ldots, R-1$; each part of the GOP between these frames is defined as a subGOP;

(ii) repeat for each sub-GOP: take $R-1$ reference frames uniformly distributed in the sub-GOP and define accordingly $R$ remaining sub-GOPs.

Figure 6 then corresponds to $N=15$ and $R=2$ for each level. Another illustration is given in Figure 7 for a GOP of 19 frames, with subframe rate $R=3$ and three temporal levels.

In such generalizations, note that for values of $N$ different from $2^{L}-1$, that we call “irregular" $N$ values, many different decompositions can be proposed that will have similar performances. As a consequence, when considering such irregular values of $N$, it is recommended to consider adaptation of the generalized pattern based on regular repartition of reference frames at each level.

To illustrate the advantage of this Zigzag scalable structure, we introduce other GOP reorganizations that correspond to structures with smaller gaps between the frames at the first level of importance. Two such decompositions that can be seen as variations of the Zigzag shuffling are considered. The first, called the "Christmas Tree" decomposition, is obtained as follows:

(i) select a first reference frame (the intraframe) placed at the median position in the GOP, where the median

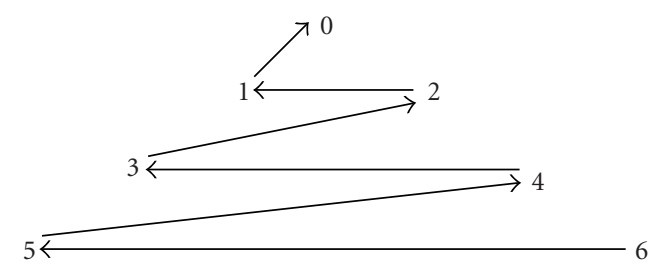

Figure 8: Christmas Tree configuration, GOP size $=7$.

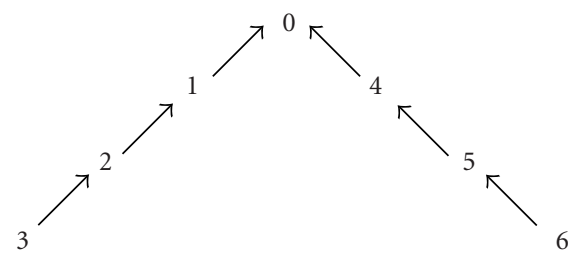

Figure 9: Mirror configuration, GOP size $=7$.

temporal index is median $=\left\lfloor\left(\mathrm{GOP}_{\text {size }}+1\right) / 2\right\rfloor$, and define the two parts separated by the median as subGOPs;

(ii) repeat alternatively for each sub-GOP (e.g., by beginning with the left sub-GOP): use the frame closest to the median one as reference and remove it from the sub-GOP frame set.

The "Mirror" decomposition is obtained as follows:

(i) select a first reference frame (the intraframe) and place it at the median position in the GOP, where the median index is median $=\left\lfloor\left(\mathrm{GOP}_{\text {size }}+1\right) / 2\right\rfloor$, and define the parts separated by the median frame as sub-GOPs;

(ii) repeat for each sub-GOP: use the frame closest to the median one as reference and define the set of remaining frames as a new sub-GOP.

Illustrated, respectively, in Figures 8 and 9 for $N=7$, these Christmas Tree and Mirror configurations will provide better results in terms of compression as with these configurations, each frame is at closer distance to its main reference than in Zigzag. However, this is obtained at the cost of a less efficient temporal scalability. Indeed, if the last refinement levels are lost, the reconstructed sequence presents long frozen subsequences.

Note also that the Mirror configuration is somehow different from the Zigzag and Christmas Tree ones in the sense that the two sub-GOPs on each side of the intraframe are in fact independent from each other. Therefore, the Mirror configuration can be considered a first type of limited reference configurations, close to those that will be presented in Section 3.3.

\subsection{Asymmetric filtering schemes}

Let us now consider the case when two intraframes are used for the prediction of the frames in a given GOP. This configuration ensuring the temporal scalability features, that we 


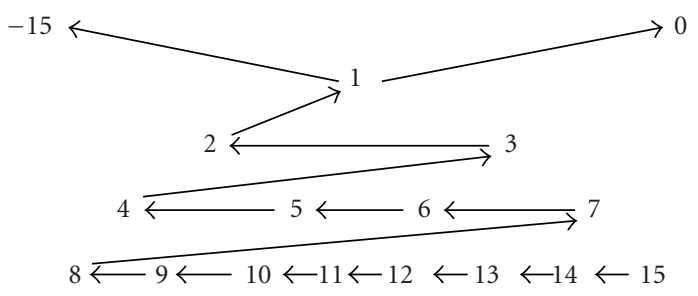

FIgure 10: Dyad configuration, GOP size $=16$.

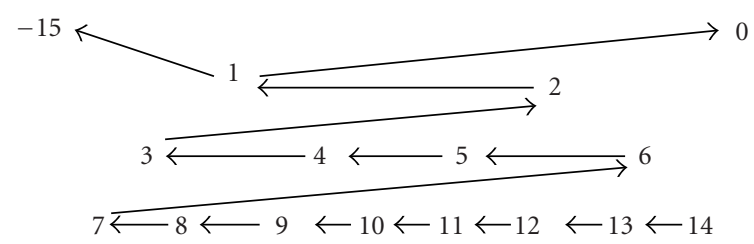

Figure 11: Generalized Dyad configuration, GOP size $=15$.

will call "Dyad" configuration, is a regular repartition pattern that corresponds to a closed-loop $2^{L}$-band filter bank, as described in Section 2, Figure 4. It can be obtained as follows:

(i) select a reference frame (the intraframe) placed at the extremity of the GOP (e.g., at the right extremity, when the other considered intraframe is the one of the previous GOP), and define the set of remaining frames as sub-GOP;

(ii) apply the Zigzag decomposition to the sub-GOP.

This is illustrated in Figure 10 for $N=16$. A generalization can here be done, following the generalization of the Zigzag decomposition principle. As an example, we give in Figure 11 a decomposition pattern for $N=15$.

In this Dyad configuration, the dependency on the intraframes is even more important, as any error in a frame at the first level leads to errors in two consecutive GOPs. In turn, the compression efficiency is better than that of the Zigzag decomposition, as the number of high quality references is higher.

\subsection{Limited references filtering schemes}

Due to some practical limitations, coming either from the use of given levels [10] in the standard profiles or from practical implementation limitations, the configurations presented in the previous sections may not be realistic, as the codec may not be allowed to use up to 16 references in its prediction algorithm. As such, it becomes important to propose decompositions with a limited number of reference frames, this number being intimately linked to the total memory necessary to implement the encoding and decoding process. Naturally, such a limitation leads to some degradation in terms of compression efficiency, but it first meets the requirements of any level for any profile in H.264/MPEG-4 AVC (hence also any practical implementation), and second it en-

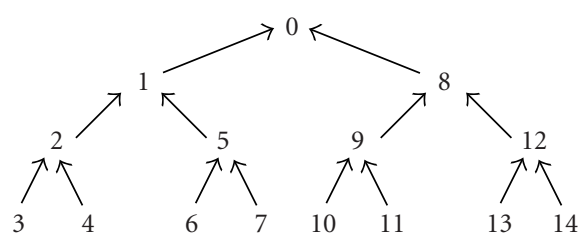

Figure 12: Tree configuration, GOP size $=15$.

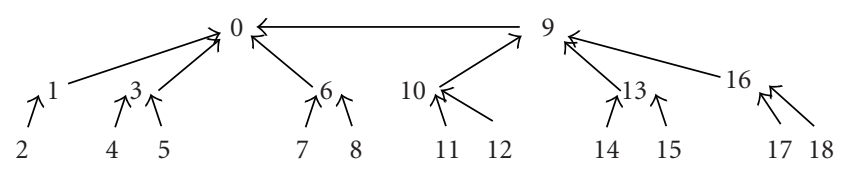

Figure 13: Tree configuration, GOP size $=19$.

sures that the error propagation can be further reduced in erroneous environments.

Considering first the unidirectional schemes presented in Section 3.1, the reduction of the number of reference frames can be done by imposing that a frame can only use reference frames from the upper levels. This "Tree" configuration (illustrated in Figure 12 for $N=15$ and in Figure 13 for $N=19$ and $R=3$ ) is obtained as follows:

(i) apply the Zigzag decomposition to assign to each frame its corresponding level of refinement;

(ii) repeat for each frame: choose as reference frame (or father) the closest one between those in the refinement level immediately above. When two frames can be equivalently chosen as reference, select the one that is the closest to its own father, and so on. If no discrimination can be done, choose, for instance, the one closest to the intraframe.

In this Tree configuration, the dependencies between frames are clearly reduced, which will be a major advantage in a noisy environment, as errors occurring at lower refinement levels will be less likely to propagate.

Considering now the Dyad scheme and its generalization, as presented in Section 3.2, the reduction of the number of references can be done similarly to the symmetric case by imposing again that a frame can only use reference frames from the upper levels. This "Limited Dyad" configuration is obtained as follows:

(i) apply the Dyad decomposition to assign to each frame its corresponding level of refinement;

(ii) repeat for each frame: choose as reference frame (or father) the closest one from those in the refinement level immediately above. When two frames can be equivalently chosen as reference, select the one that is the closest to its own father, and so on. If no discrimination can be done, choose, for instance, the one closest to the intraframe.

Limited Dyad configuration is illustrated in Figures 14 and 15 for $N=16$ and $N=15$, respectively. The limitation 


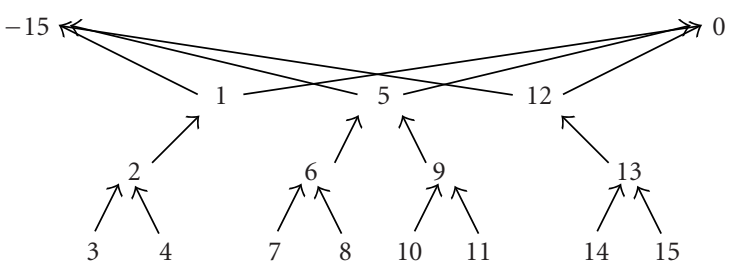

FIgURe 14: Limited Dyad configuration, GOP size $=16$.

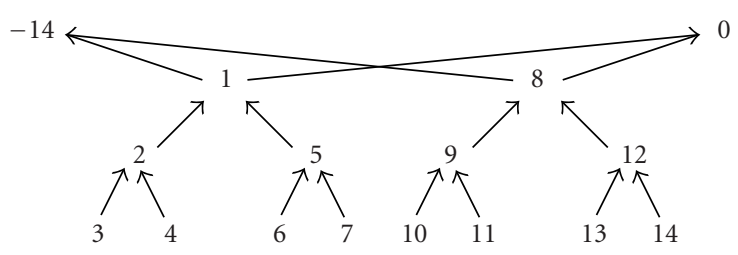

Figure 15: Limited Dyad configuration, GOP size $=15$.

on the number of reference frames clearly reduces the dependencies between frames, which will ensure that error propagation is limited in an error-prone environment. However, as for the other configurations, the loss of intraframes will affect all the frames that reference them.

\section{IMPLEMENTATION DETAILS}

The purpose of the schemes presented in the previous sections is to introduce temporal scalability within an a priori nonscalable configuration, such as the one provided by the H.264/MPEG-4 AVC codecs. The scheme shuffles the frames in a GOP to distribute them as regularly as possible.

The practical implementation of the different schemes presented in Section 3 is easily done in a standard compatible codec based on the consideration that two different frame numbering solutions do exist in the H.264/MPEG-4 AVC standard. The first, frame_num, corresponds to the decoding order of access units, but does not necessarily indicate the final display order that the decoder will use. The second, POC or picture order count, corresponds to the display order of the decoded frames (or fields) that will be used by the decoder for the display order. Considering now the number of reference frames to be used, here again the practical implementation is quite easily managed thanks, in the case of nonlimited models, to the existence of a reference buffer of up to 16 different frames for any P-slice, and in the case of limited models, to the existence of memory management standardised functions that can be used to remove given frames from the reference buffer or mark given frames not to be used as reference. The only drawback of this scheme is that the shuffling operation introduces a delay and the necessity of frame buffering, both at the encoder and the decoder sides.

As presented in Section 3, the most important frames, corresponding to those decoded from the lowest frame rates, can be regularly distributed along the time frame. The intervals between those most important frames are then filled with less important ones, that are decoded only at higher frame rates. A temporal scalability enhanced H.264/MPEG4 encoder can thus be implemented by first performing a rearrangement of the frames according to their encoding order before the source encoder, and then by classical H.264/MPEG-4 AVC encoding.

The advantage of being able to define different scalable configurations at the encoder side, while not needing to transmit any supplementary information to the decoder or predefining the said configuration during an initialisation phase, is that the chosen configuration can adapt either to the sequence actually being transmitted or to the channel conditions. As an example, transmitting over an erroneous channel may favor limited reference schemes, in order to avoid error propagation. Also, the choice of the frame shuffling pattern can be made based on GOP particularities. For instance, to better take into account some scene cuts, the frames corresponding to such changes will be coded with higher quality (the choice of the pattern will then be made such that they are placed at a low level of temporal resolution) and consequently ensure high rendering quality thanks to a better adaptivity of the codec. This GOP analysis and shuffling may lead however to a delay in sequence transmission, which needs to be compatible with the time constraints of the application. Finally, in a less adaptive mode, the choice of the configuration can be made based on the capabilities of the encoder and the decoder, in particular when they are implemented on low-memory/CPU platforms. The simulation chain used to obtain and test the scalability features is presented in Figure 16. The shuffling operation is applied directly on the video sequence to be encoded by means of an interleaver (denoted by $\Pi$ in the figure), before the standard H.264/MPEG-4 AVC encoding process which is only modified to the extent of inserting knowledge of the used shuffling table, corresponding to the different scalability configurations presented, to permit the insertion of the correct display order values in the POC fields. The fully compliant H.264/MPEG-4 AVC code stream can then be sent over the transmission channel, which can be error-prone, as in case of transmission over wireless links, or error-free, as in case of transmission with an efficient forward error correction/automatic repeat-request mechanism.

\section{SIMULATION RESULTS}

The simulations used the joint verification model (JM) version 8.4 [11], with some modifications, to ensure that the number of frames that can be used as reference corresponds to the actual number of decomposition patterns. Indeed, some of the proposed patterns need more reference frames than the maximum number implemented by JM8.4.

The average PSNR values, derived as the average of MSE values over the whole sequence, for "Foreman," "Mobile", and "Akiyo" reference sequences (QCIF, $15 \mathrm{~Hz}, M=7$ ) are given in Table 1 for different unidirectional decompositions and regular GOP sizes equal to 7 or 15 . In each case, the quantization parameters have been adjusted to yield a target bit rate of $64 \mathrm{kbps}$ or $128 \mathrm{kbps}$. 


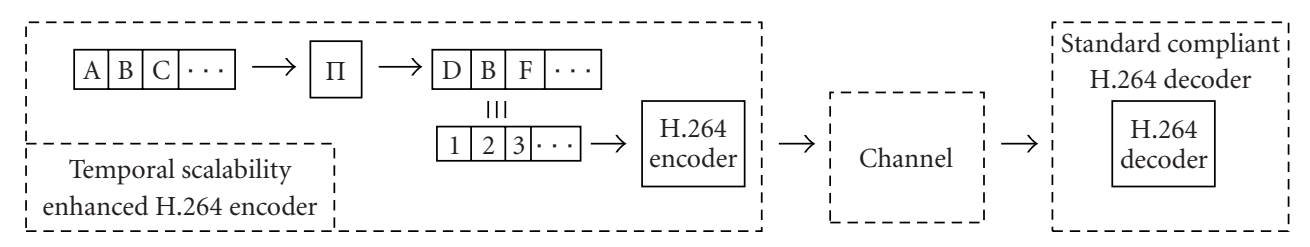

FIgURE 16: Simulation chain. The block denoted by $\Pi$ corresponds to the interleaver.

TAble 1: Average PSNR (over the entire sequence) at $64 \mathrm{kbps}$ and $128 \mathrm{kbps}$ for different configurations of the symmetric hierarchical subband tree H.264/MPEG-4 AVC codec for QCIF $15 \mathrm{~Hz}$ video sequences and GOP size $2^{L}-1$.

\begin{tabular}{c|cc|cc}
\hline Sequence & Bit rate & Configuration & \multicolumn{2}{|c}{ Av. PSNR (dB) } \\
& GoPs size 7 & GOP size 15 \\
\hline Akiyo & 64 & Normal & 40.19 & 43.09 \\
Akiyo & 64 & Mirror & 40.41 & 43.36 \\
Akiyo & 64 & Christ. Tree & 40.33 & 43.15 \\
Akiyo & 64 & Tree & 40.32 & 43.34 \\
Akiyo & 64 & Zigzag & 40.32 & 43.25 \\
Foreman & 64 & Normal & 32.19 & 33.35 \\
Foreman & 64 & Mirror & 32.54 & 33.71 \\
Foreman & 64 & Christ. Tree & 32.36 & 33.38 \\
Foreman & 64 & Tree & 32.48 & 33.58 \\
Foreman & 64 & Zigzag & 32.28 & 33.28 \\
Mobile & 128 & Normal & 27.94 & 29.66 \\
Mobile & 128 & Mirror & 28.32 & 30.30 \\
Mobile & 128 & Christ. Tree & 28.26 & 29.96 \\
Mobile & 128 & Tree & 28.30 & 30.12 \\
Mobile & 128 & Zigzag & 28.27 & 30.03 \\
\hline
\end{tabular}

It can be observed that the scalability feature is obtained in each case with small (less than $1 \%$ in the worst case, compared with the mirror configuration - at the tested bit rates, this is independent of the bit rate, but it may slightly depend on the sequence characteristics) or no quality degradation. This confirms the advantage of placing the intraframe at the median position of the GOP (in the display order), which reduces the maximum distance between a predicted frame and its reference. By comparing the differences between different configurations (which are quite small), the advantage of choosing the configuration according to the actual transmission conditions, that is to say to adapt the configuration choice either to the transmission channel, to the sequence actually been transmitted, or to the encoder or decoder capacities, as mentioned in Section 4, becomes obvious. Still, it can be observed that the Tree and Mirror configurations obtain here the best performance among all configurations. This can be partly explained by a particularity of the H.264/MPEG4 AVC codec syntax, which relies on variable-length codes for indicating the considered reference frames. The Tree and Mirror patterns, ensuring that frames mostly use as reference the closest one in decoding order, have then an advantage when compared to the others.
TABle 2: Average PSNR at $64 \mathrm{kbps}$ and $128 \mathrm{kbps}$ for the different configurations of the Dyad hierarchical subband tree H.264/MPEG4 AVC codec for QCIF $15 \mathrm{~Hz}$ video sequences and GOP size $2^{L}$.

\begin{tabular}{c|ccc}
\hline Sequence & $\begin{array}{c}\text { Bit rate } \\
(\mathrm{kbps})\end{array}$ & Configuration & $\begin{array}{c}\text { Av. PSNR (dB) } \\
\text { GOP size 16 }\end{array}$ \\
\hline Akiyo & 64 & Dyad & 43.59 \\
Akiyo & 64 & Limited Dyad & 43.85 \\
Foreman & 64 & Dyad & 33.58 \\
Foreman & 64 & Limited Dyad & 33.79 \\
Mobile & 128 & Dyad & 30.21 \\
Mobile & 128 & Limited Dyad & 30.52 \\
\hline
\end{tabular}

Results obtained for the same three sequences and same target bit rates for different asymmetric decompositions and regular GOP size equal to $2^{L}$ are presented in Table 2, where the average PSNR is computed over the entire sequence. Comparing the two asymmetric configurations, it can be observed that, like for the symmetric ones, the limited version performs better than the Dyad one, based on Zigzag decomposition, for the same syntactical reasons. Based on this observation, we can now compare the results for a GOP size of 16 with those obtained for symmetric decompositions and GOPs of size 15. One can observe a quality gain of 0.1 to $0.5 \mathrm{~dB}$. Yet, this is obtained at the cost of a higher dependency on the intraframes, which again highlights the importance of choosing the hierarchical configuration in error-prone environments according to the actual transmission conditions, and not only based on pure average PSNR considerations.

A second set of simulations have been conducted in an erroneous context, to observe the impact of transmission errors in various configurations. In our experiments, we selected a scenario where one frame in the GOP (the sixth frame when the first frame of the GOP is frame 0 ) is impaired (completely black) at the decoder. ${ }^{3}$

We observed the corresponding PSNR evolution of the whole GOP. Figures 17, 18, and 19 present the PSNR evolution for Foreman QCIF, $15 \mathrm{~Hz}, 64 \mathrm{kbps}$, GOP size $=15$ for Normal, Zigzag, and Tree configurations in the case of loss in information in the 6 th frame in the GOP (i.e., frame number 5 in the encoding order) which appears in different scalable modes in the enhancement level. In these figures, the $x$-axis

\footnotetext{
${ }^{3}$ A black frame at the decoder can be obtained if the NAL header is received, but it is incorrect. Such case can happen when bit errors are present.
} 


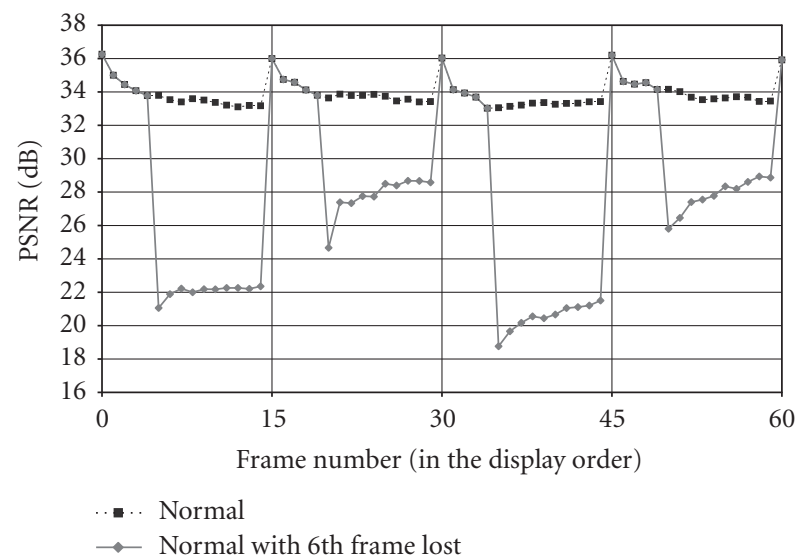

FIGURE 17: Foreman PSNR evolution for the Normal configuration in an error-free and an erroneous environment (every 6th coded frame impaired). The $x$-axis indicates the frame number in the output (display) order.

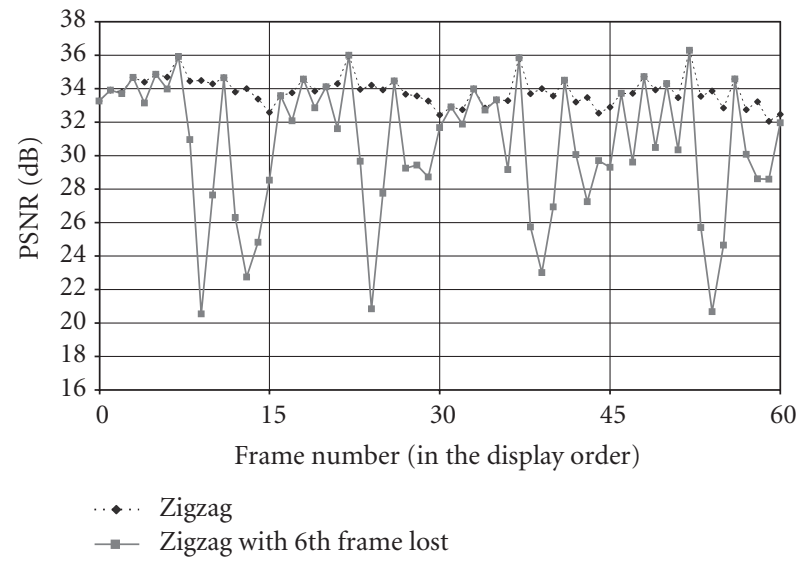

FIGURE 18: Foreman PSNR evolution for the Zigzag configuration in an error-free and an erroneous environment (every 6th coded frame impaired). The $x$-axis indicates the frame number in the output (display) order.

indicates the frame number in the output (display) order. As foreseen from the results presented in Table 1, the three configurations have similar results in error-free environments. However, this changes greatly when errors occur. As a matter of fact, the degradation is quite noticeable for the Normal configuration, due to the error propagation from the erroneous frame to the end of the GOP. The Zigzag configuration presents the same number of frames affected by the error propagation, but the frame shuffling reduces the impact of errors due to the fact that most of those frames are partly predicted from correct ones. Finally, the Tree configuration limits the error propagation to a small set of frames, which in counterpart are more deeply degraded due to the fact that when compared to Normal case they rely on only a small set of reference frames, with one of their main reference being impaired.

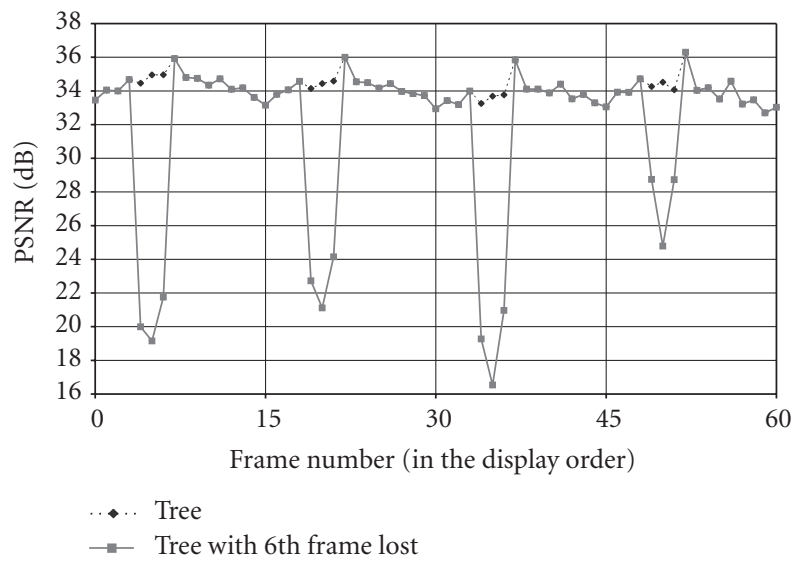

FIGURE 19: Foreman PSNR evolution for the Tree configuration in an error-free and an erroneous environment (every 6th coded frame impaired). The $x$-axis indicates the frame number in the output (display) order.

We conducted informal subjective evaluation of the decoded sequences affected by frame loss. The corresponding visual results obtained for one entire GOP are presented in Figure 20 for the Normal configuration, in Figure 21 for the Zigzag one, and in Figure 22 for the Tree one. The degradation due to the impaired frame is clearly more annoying in the Normal case as it leads to the degradation of the entire second part of the GOP, whereas it is quite acceptable in the Zigzag case, where the degradations are less distinguishable. They are even less important in the "Tree" case, where only three frames are degraded. The concealment in this later case is very easy, the impaired frame (6th in the display order) being restored by frame copy from its main reference (and, due to this, looking visually "good," even though it is not correct, i.e., it is not equivalent to the original frame) and the two frames depending on it (5th and 7th in the display order) being the only ones predicted from an erroneous frame (more sophisticated concealment techniques can also be applied). The PSNR results for these three frames are quite low, but visually (see Figure 22) even the simple concealment technique we used (frame copy from the main reference) provides very satisfactory results.

Finally, let us illustrate the advantage of adapting the scalable pattern based on transmission conditions, as mentioned in Section 4 for the case when a back channel is available. Considering the case of a wireless channel such as the GSM or UMTS ones, where errors often appear in bursts, the video transmission is confronted with time intervals when the channel is error-free and others where the channel is erroneous. In practice, based on simulation results presented in Tables 1 and 2, the pattern recommended for error-free channels can be Limited Dyad configuration, which offers the best PSNR of all configurations. Now, when considering noisy transmissions, the impact of loosing an intraframe is more dramatic on bidirectional configurations as the intra is used for prediction over two GOPs. As such, one can recommend the following efficient adaptation pattern: 

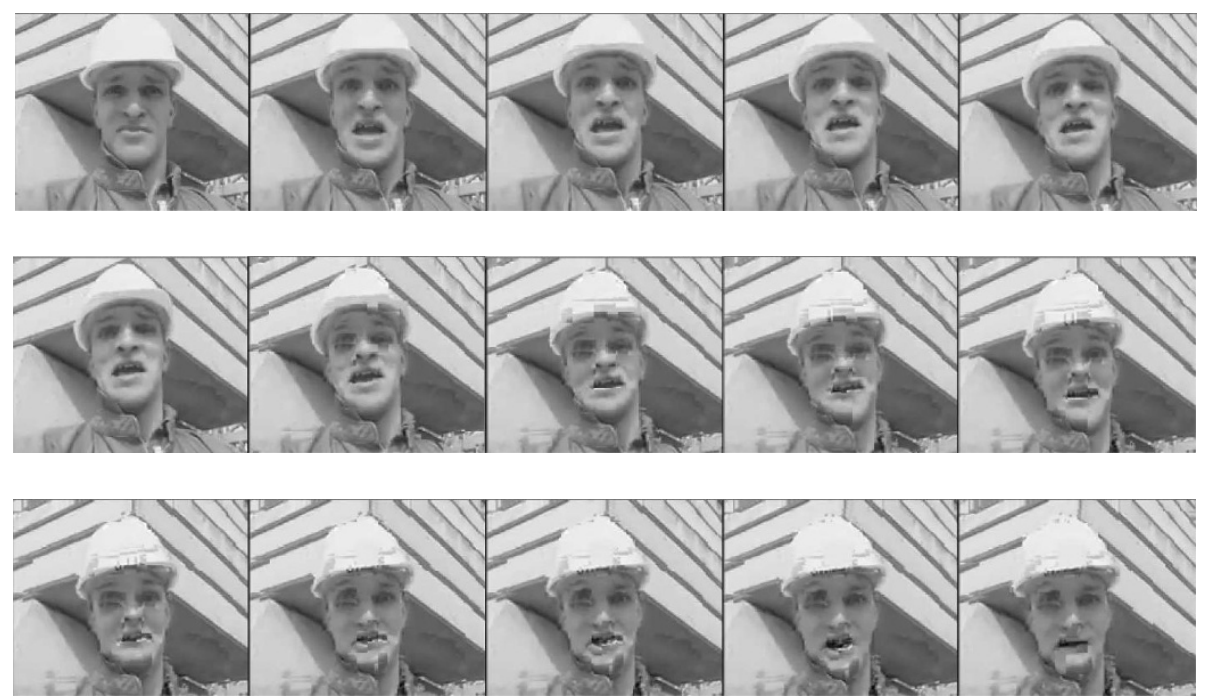

FIgUre 20: Visual results over a GOP $(N=15)$ in an erroneous environment for the Normal configuration, Foreman sequence (6th frame impaired).
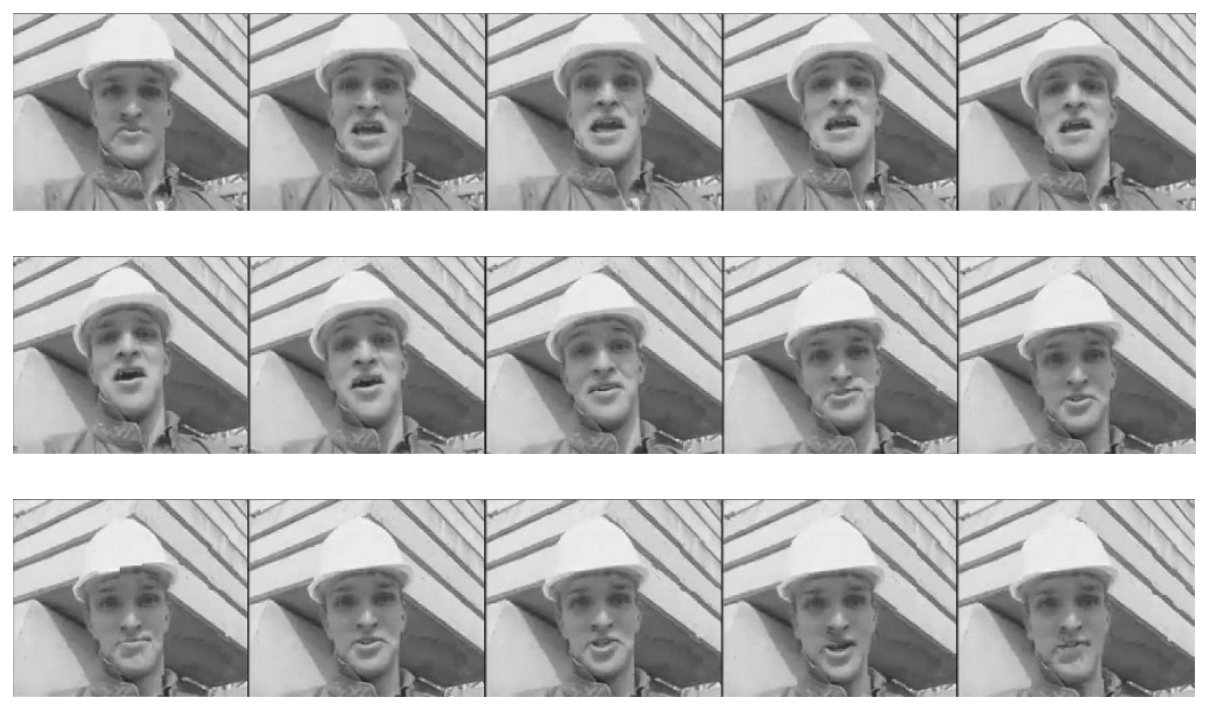

FIgURe 21: Visual results over a GOP $(N=15)$ in an erroneous environment for the Zigzag configuration (6th frame impaired).

(i) use Limited Dyad configuration by default;

(ii) when detecting at the receiver side that an intraframe has been impaired, inform the encoding side by the back channel and suggest to select a symmetric configuration, for instance, Tree, and use it up until a sufficient number of frames have been received without errors.

Figure 23 illustrates the results obtained when comparing the use of a nonadaptive Limited Dyad configuration over several GOPs with the use of the adaptive method proposed above, where the intraframe of the second GOP has been im- paired (i.e., the 17th frame in encoding order and the 32th one in decoding order). The advantage of going back for one GOP to Tree configuration is obvious, while Limited Dyad remains the best choice when the channel is error-free.

\section{CONCLUSIONS}

In this paper, we have introduced a general $M$-band filter bank framework for adaptive motion-compensated temporal filtering and have shown how different temporal scalable solutions can be derived from it in an H.264/MPEG-4 AVC compliant manner. The proposed configurations have 

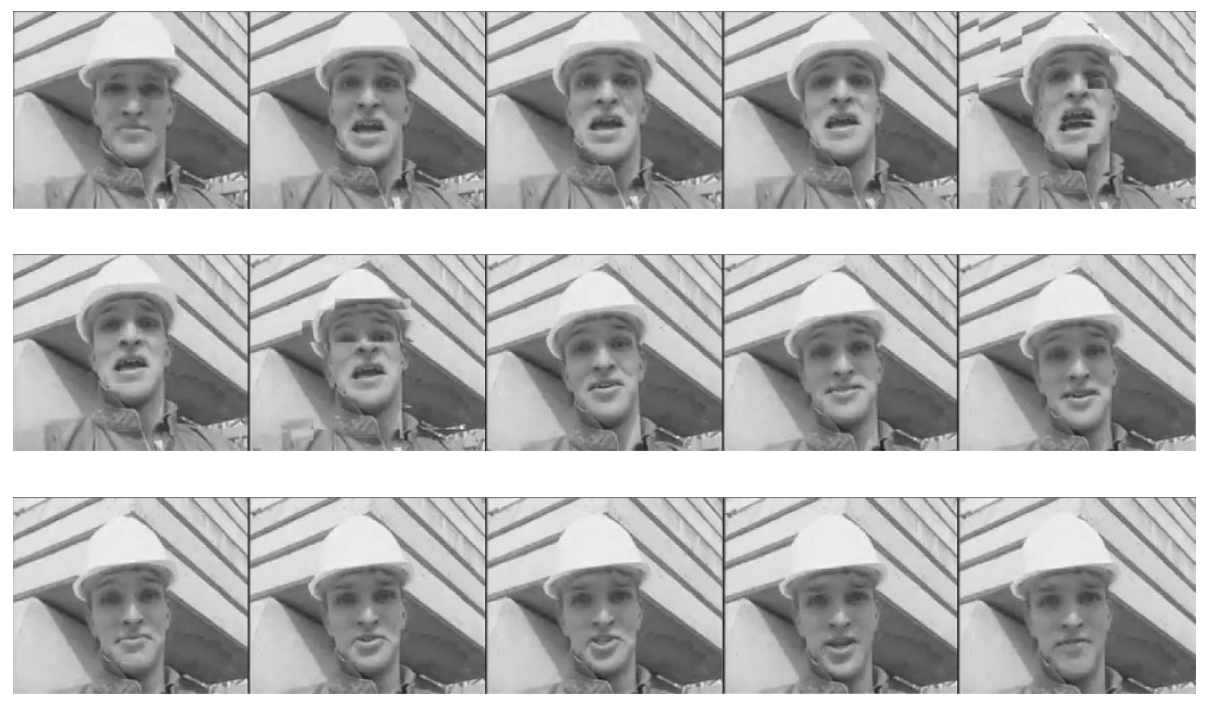

FIgURE 22: Visual results over a GOP $(N=15)$ for the Tree configuration in an erroneous environment (6th frame impaired).

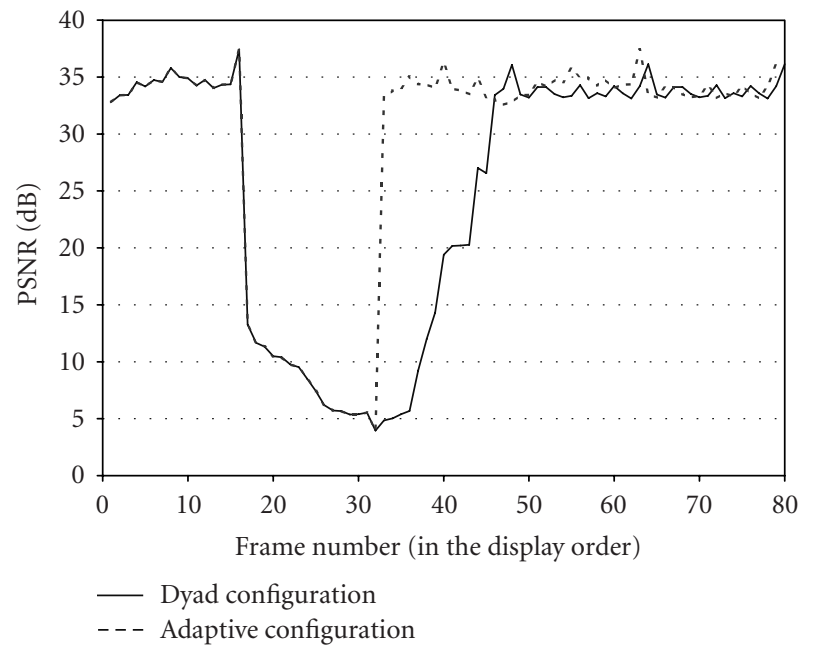

FIGURE 23: Foreman PSNR evolution comparison in noisy environment: Dyad configuration versus Adaptive mode.

been compared in error-free and error-prone environments and the advantage provided by scalability in terms of robustness has been shown. By analysing the dependencies between frames in these configurations, one can predict not only the error propagation, but also the impact of the sequence features on the ability to perform error concealment.

\section{ACKNOWLEDGMENT}

This work was partially supported by the European Community through project IST-FP6-001812 PHOENIX and project IST-FP6-1-507113 DANAE.

\section{REFERENCES}

[1] Joint Video Team (JVT) of ISO/IEC MPEG ITU-T VCEG, Draft ITU recommendation and final draft international stan- dard of joint video specification (ITU-T Rec. H.264/ISO/IEC 14 496-10 AVC), Doc JVT-G050r1, Geneva, Switzerland, May 2003.

[2] J.-R. Ohm, Registered Responses to the Call for Proposals on Scalable Video Coding. ISO/IEC JTC1/SC29/WG11 MPEG, doc. M10569, proposal S16, Munich, Germany, March 2004.

[3] L. Blaszak, M. Domanski, A. Luczak, and S. Mackowiak, "AVC video coders with spatial and temporal scalability," in Proceedings of Picture Coding Symposium (PCS '03), pp. 41-47, SaintMalo, France, April 2003.

[4] H. Schwarz, D. Marpe, and T. Wiegand, Subband extension for H.264/AVC, Doc JVT-K023, Munich, Germany, March 2004.

[5] J.-R. Ohm, Multimedia Communication Technology: Representation, Transmission and Identification of Multimedia Signals, Springer, Berlin, Germany, 2004.

[6] G. Pau, C. Tillier, B. Pesquet-Popescu, and H. Heijmans, "Motion compensation and scalability in lifting-based video coding," Signal Processing: Image Communication, vol. 19, no. 7, pp. 577-600, 2004, special issue on wavelet video coding.

[7] J. Xu, Z. Xiong, S. Li, and Y.-Q. Zhang, “Three-dimensional embedded subband coding with optimized truncation (3DESCOT)," Applied and Computational Harmonic Analysis, vol. 10, no. 3, pp. 290-315, 2001.

[8] C. Bergeron, C. Lamy-Bergot, and B. Pesquet-Popescu, "Adaptive $M$-band hierarchical filterbank for compliant temporal scalability in H.264 standard," in Proceedings of IEEE International Conference on Acoustics, Speech, and Signal Processing (ICASSP '05), vol. 2, pp. 69-72, Philadelphia, Pa, USA, March 2005.

[9] B. Pesquet-Popescu and V. Bottreau, "Three-dimensional lifting schemes for motion compensated video compression," in Proceedings of IEEE International Conference on Acoustics, Speech, and Signal Processing (ICASSP '01), vol. 3, pp. 17931796, Salt Lake City, Utah, USA, May 2001.

[10] A. Luthra and P. N. Topiwala, "Overview of the H.264/AVC video coding standard," in Applications of Digital Image Processing XXVI, A. G. Tescher, Ed., vol. 5203 of Proceedings of SPIE, pp. 417-431, San Diego, Calif, USA, August 2003. 
[11] Joint verification model for H.264 (JM 8.4), July 2004, http:// iphome.hhi.de/suehring/tml.

C. Bergeron was born in Oyonnax, France, in 1978. He received the Electrical Engineering degree from the Ecole Superieure d'Ingenieurs de Nice Sophia-Antipolis (ESINSA) in 2001, and the Master's degree (Diplome d'Etudes Approfondies) in signal and image processing from the Ecole Doctorale de Nice Sophia-Antipolis in 2003. He is currently a Ph.D. student at the Image and Signal Processing Department

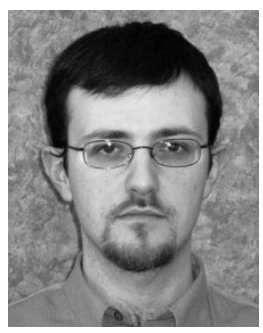
of Ecole Nationale Superieure des Telecommunications (ENST), in collaboration with THALES Communications. His research interests include video source coding, H.264, error protection techniques, and joint source and channel decoding.

C. Lamy-Bergot was born in Vernon, France, in 1972. She received in 1996, both the Electrical Engineering degree and Master's degree (Diplôme d'Etudes Approfondies) from the Ecole Nationale Superieure des Télécommunications (ENST), Paris, France, and the Ph.D. degree in 2000. She was with Philips Research France, from 2000 to 2002, where she worked as a Research Scientist on joint source and channel coding techniques. She joined THALES Communications as a Senior Scientist in digital communications in September 2002 to work in the Signal Processing and Multimedia Department. She has participated in national and European projects such as RNRTVIP, IST-2KAN, and IST-NEWCOM and is currently leading IST PHOENIX project. Her fields of interest include iterative decoding techniques, space time codes, high-efficiency modulations, error correction codes, unequal error protection, soft output decoding, and joint source and channel coding techniques.

G. Pau was born in Toulouse, France, in 1977, and received the M.S. degree in signal processing in 2000 from Ecole Centrale de Nantes. From 2000 to 2002, he worked as a Research Engineer at Expway where he actively contributed to the standardization of the MPEG-7 binary format. He is currently a Ph.D. candidate in the Signal and Image Processing Department, ENSTTelecom, Paris. His research interests include subband video coding, motion-compensated temporal filtering, and adaptive nonlinear wavelet transforms.

B. Pesquet-Popescu received the Engineering degree in telecommunications from the "Politehnica" Institute, Bucharest, in 1995, and the Ph.D. degree from the Ecole Normale Supérieure de Cachan in 1998. In 1998, she was a Research and Teaching Assistant at Université Paris XI, and in 1999 she joined Philips Research France, where she worked for two years as a Research Scientist in scalable video coding. Since Octo-

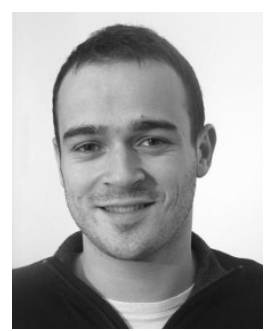

ber 2000, she has been an Associate Professor in multimedia at the Ecole Nationale Supérieure des Télécommunications (ENST). Her current research interests are in scalable and robust video coding, adaptive wavelets, and multimedia applications. EURASIP gave her a "Best Student Paper Award" in the IEEE Signal Processing Workshop on Higher-Order Statistics in 1997, and in 1998, she received a "Young Investigator Award" granted by the French Physical Society. She holds 20 patents in wavelet-based video coding and has authored more than 80 book chapters, journal and conference papers in the field. 